CVIA

REVIEW ARTICLE

pISSN 2508-707X / elSSN 2508-7088 https://doi.org/10.22468/cvia.2017.00066 CVIA 2017;1(3):156-165

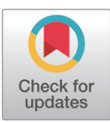

\section{Multimodality Appropriate Use Criteria for Noninvasive Cardiac Imaging: Expert Consensus of the Asian Society of Cardiovascular Imaging}

\author{
ASCI Practice Guideline Working Group; Kyongmin Sarah Beck ${ }^{1 *}$, \\ Jeong A Kim²*, Yeon Hyeon Choe', Sim Kui Hian', John Hoe', \\ Yoo Jin Hong ${ }^{6}$, Sung Mok Kim ${ }^{3}$, Tae Hoon $\mathrm{Kim}^{7}$, Young Jin Kim ${ }^{6}$, \\ Yun Hyeon $\mathrm{Kim}^{8}$, Sachio Kuribayashi ${ }^{9}$, Jongmin Lee ${ }^{10}$, \\ Lilian Leong ${ }^{11}$, Tae-Hwan Lim² ${ }^{12}$ Bin Lu' ${ }^{13}$, Jae Hyung Park ${ }^{14}$, \\ Hajime Sakuma ${ }^{15}$, Dong Hyun Yang ${ }^{12}$, Tan Swee Yaw ${ }^{16}$, \\ Yung-Liang Wan ${ }^{17}$, Zhaoqi Zhang ${ }^{18}$, \\ Shihua Zhao ${ }^{13}$, Hwan Seok Yong ${ }^{19}$
'Department of Radiology, Seoul St. Mary's Hospital, College of Medicine, The Catholic University of Korea, Seoul, Korea
2Department of Radiology, Ilsan Paik Hospital, Inje University College of Medicine, Goyang, Korea ${ }^{3}$ Department of Radiology, Samsung Medical Center, Sungkyunkwan University
School of Medicine, Seoul, Korea
${ }^{5}$ Department of Radiology, Mount Elizabeth Hospital, Singapore, Singapore
${ }^{6}$ Department of Radiology, Severance Hospital, Yonsei University College of Medicine,
Seoul, Korea
${ }^{7}$ Department of Radiology, Gangnam Severance Hospital, Yonsei University College of Medicine, Seoul, Korea
${ }^{8}$ Department of Radiology, Chonnam National University Hospital, Gwangju, Korea 'Department of Diagnostic Radiology, Keio University, Tokyo, Japan
${ }^{10}$ Department of Radiology, Kyungpook National University Hospital, Daegu, Korea
${ }^{11}$ Department of Radiology, Hong Kong College of Radiologists, Hong Kong, China
${ }^{12}$ Department of Radiology, Asan Medical Center, University of Ulsan College of Medicine, Seoul, Korea
${ }^{13}$ Department of Radiology, Fuwai Hospital, Chinese Academy of Medical Science \&
Peking Union Medical College, National Center for Cardiovascular Diseases, Beijing, China
${ }^{14}$ Department of Radiology, Myongji Hospital, Goyang, Korea
${ }^{15}$ Department of Radiology, Mie University Hospital, Mie, Japan
${ }^{16}$ Department of Cardiology, National Heart Centre Singapore, Singapore, Singapore
${ }^{17}$ Department of Medical Imaging and Intervention, Chang Gung Memorial Hospital at Linkou, Institute for Radiological Research, College of Medicine, Chang Gung University, Taoyuan, Taiwan ${ }^{18}$ Department of Radiology, Beijing Anzhen Hospital, Capital Medical University, Beijing, China
${ }^{19}$ Department of Radiology, Korea University Guro Hospital, Korea University College of Medicine, Seoul, Korea \\ ${ }^{4}$ Department of Cardiology, Sarawak General Hospital Heart Centre, Sarawak, Malaysia
}

In 2010, the Asian Society of Cardiovascular Imaging (ASCI) provided recommendations for cardiac CT and MRI, and this document reflects an update of the $2010 \mathrm{ASCl}$ appropriate use criteria (AUC). In 2016, the ASCI formed a new working group for revision of AUC for noninvasive cardiac imaging. A major change that we made in this document is the rating of various noninvasive tests (exercise electrocardiogram, echocardiography, positron emission tomography, single-photon emission computed tomography, radionuclide imaging, cardiac magnetic resonance, and cardiac computed tomography/angiography), compared side by side for work.

(a) This is an Open Access article distributed under the terms of the Creative Commons Attribution Non-Commercial License (http://creativecommons.org/licenses/by-nc/4.0) which permits unrestricted non-commercial use, distribution, and reproduction in any medium, provided the original work is properly cited. 
their applications in various clinical scenarios. Ninety-five clinical scenarios were developed from eight selected pre-existing guidelines and classified into four sections as follows: 1) detection of coronary artery disease, symptomatic or asymptomatic; 2) cardiac evaluation in various clinical scenarios; 3) use of imaging modality according to prior testing; and 4) evaluation of cardiac structure and function. The clinical scenarios were scored by a separate rating committee on a scale of 1-9 to designate appropriate use, uncertain use, or inappropriate use according to a modified Delphi method. Overall, the AUC ratings for CT were higher than those of previous guidelines. These new AUC provide guidance for clinicians choosing among available testing modalities for various cardiac diseases and are also unique, given that most previous AUC for noninvasive imaging include only one imaging technique. As cardiac imaging is multimodal in nature, we believe that these AUC will be more useful for clinical decision making.

Key words Appropriate use criteria · Multimodality · Noninvasive cardiac imaging.

\section{INTRODUCTION}

Noninvasive cardiac imaging procedures provide essential information for the detection, diagnosis, and management of cardiovascular diseases and serve a vital role in risk assessment and clinical decision making. The range of diagnostic tools used to evaluate cardiovascular disease has expanded over the past decade; in particular, computed tomography (CT) and magnetic resonance (MR) have emerged as alternatives to echocardiography, exercise electrocardiography (ECG), and invasive angiography.

Guidelines developed in the United States and Europe are often not applicable in Asian countries because of differences in healthcare systems, medical expenses, body habitus, and disease prevalence between Asian and Western countries. For this reason, the Asian Society of Cardiovascular Imaging (ASCI) separately developed ASCI appropriate use criteria (AUC) for cardiac CT and MR in 2010. Since the introduction of ASCI AUC in 2010, there has been further accumulation of scientific evidence and advances in imaging technology.

Currently, there are many guidelines published by different cardiovascular societies led by various expert groups; as a consequence, there are various AUC for different modalities and diseases and from different countries. The American College of Cardiology Foundation (ACCF), along with key specialty and subspecialty societies, published AUC for cardiac CT and cardiac MR (CMR) in 2006 [1]. In 2010, AUC for CT were published by the Society of Cardiovascular Computed Tomography (SCCT) as well as the ASCI $[2,3]$. More recently, in 2015, the Korean Society of Radiology (KSR) also published AUC for CT [4]. AUC for CMR were also published by the ASCI in 2010 [5] and by the KSR in 2015 [6]. As for radionuclide imaging (RNI), the ACCF, along with key specialty and subspecialty societies, published AUC in 2009 [7], although they were limited to coronary artery disease only. Moreover, the ACCF and key specialty and subspecialty societies published AUC for echocardiography in 2011 [8]. There are also many guidelines for specific clinical scenarios, such as for appropriate utilization of various cardiac imaging modalities in the diagnosis and treatment of heart failure $[9,10]$, hypertrophic cardiomyopathy [11], and stable ischemic heart disease $[12,13]$. However, few guidelines encompass various clinical scenarios, and to the best of our knowledge, there have been no multimodality AUC in Asia. Thus, we believe that multimodality AUC for different clinical scenarios would be relevant as an update for the previous ASCI CT and MR guidelines. The purpose of this document is to delineate the appropriate use of various noninvasive testing modalities for the diagnosis and evaluation of heart disease, as well as to update the previous 2010 ASCI AUC for cardiac CT and MR.

\section{METHODS}

\section{Plans and approval for ASCI guideline update}

In March 2016, the need for an update of ASCI AUC was discussed at the ASCI Administration office-presidential office meeting. A steering committee was appointed among the board of directors in order to establish plans and a budget for the AUC update. In addition, a writing committee was to be appointed from the Korean members, a rating committee to be comprised of major speakers and researchers among the ASCI members, and a review committee to be comprised of previous presidents, vice-presidents, and congress presidents of the ASCI. The plan was approved at the annual ASCI meeting held in Singapore in August 2016.

The working group consisted of the following committees: 


\section{Steering committee}

Yeon Hyeon Choe, Jongmin Lee, Yun Hyeon Kim, Bin Lu, Tae Hoon Kim

\section{Writing committee \\ Young Jin Kim, Jeong A Kim, Sung Mok Kim, Kyongmin Sarah Beck, Hwan Seok Yong, Dong Hyun Yang, Yoo Jin Hong}

\section{Rating committee}

The number of technical panel members on the rating committee from each country was decided by the working group according to participation in the ASCI executive committee and ASCI annual meetings, as well as academic credentials of the investigators in the field. Thirty-three experts were nominated for the technical panel, taking into account the members' nationalities and areas of expertise, and all were approved by the working group in consensus. Twenty-two of the 33 technical panel members responded and participated in the consensus process according to the modified Delphi method.

Sung A Chang (Korea, Cardiology), Jin-Ho Choi (Korea,

Cardiology), Sang-Chol Lee (Korea, Cardiology),

Seung-Pyo Lee (Korea, Cardiology), Yeonyee Yoon (Korea,

Cardiology), Kakuya Kitagawa (Japan, Radiology and

Cardiology), Keiichiro Yoshinaga (Japan, Nuclear Medicine),

Won Jun Kang (Korea, Nuclear Medicine), Jin Chul Paeng

(Korea, Nuclear Medicine), Stephen Cheung (Hong Kong,

Radiology), Akira Kurata (Japan, Radiology),

Makoto Takamiya (Japan, Radiology), Whal Lee (Korea,

Radiology), Sang IL Choi (Korea, Radiology), Eun Ju Chun

(Korea, Radiology), Joon-Won Kang (Korea, Radiology),

Sung Min Ko (Korea, Radiology), Jung Im Jung (Korea,

Radiology), Ming-Ting Wu (Taiwan, Radiology),

Wen-Yih Tseng (Taiwan, Radiology), Wen-Jeng Lee (Taiwan,

Radiology), Masahiro Jinzaki (Japan, Radiology)

\section{Review committee}

Yeon Hyeon Choe, John Hoe, Sachio Kuribayashi, Tae-Hwan Lim, Zhaoqi Zhang, Shihua Zhao, Lilian Leong, Sim Kui Hian, Jae Hyung Park, Hajime Sakuma, Oraporn See, Tan Swee Yaw

\section{Determining the methods for establishing AUC of multimodality cardiac imaging: adaptation and consensus methodology}

Because the ASCI CT and MR AUC were last published in 2010 , we searched online databases for guidelines on noninvasive imaging published since 2010; if such guidelines were unavailable for certain modalities, we then searched for the most recently published guidelines instead. The following online da- tabases were searched: Ovid-Medline, Ovid-Embase, National Guideline Clearing, and Guideline International Network. For development of this consensus document, we reviewed pre-existing utilization guidelines from countries worldwide. Eight preexisting guidelines $[3-9,13]$ were finally selected for guideline adaptation: 1) ACCF cardiac radionuclide imaging guideline 2009 (7), 2) ASCI CMR guideline 2010 (5), 3) ASCI cardiac computed tomography angiography (CCTA) guideline 2010 (3), 4) ACCF echocardiography guideline 2011 (8), 5) ACCF multimodality guideline for stable ischemic heart disease 2013 (9), 6) ACCF cardiovascular imaging in heart failure 2013 (13), 7) Korean CMR guideline 2014 (6), 8) Korean CCTA guideline 2014 (4).

The key questions were developed by the writing committee. To establish the key questions, the writing committee reviewed the previously published guidelines for each imaging modality as well as multimodality guidelines for ischemic heart disease and heart failure. After collecting all of the existing clinical questions from the guidelines, the writing committee classified the questions into four sections as follows: 1) detection of coronary artery disease, symptomatic or asymptomatic; 2) cardiac evaluation in various clinical scenarios; 3 ) use of imaging modality according to prior testing; and 4) evaluation of cardiac structure and function. Since questions in the previous guidelines varied by imaging modality, the writing committee selected questions common to each imaging method. Questions limited to a specific imaging method were changed or combined, conforming to more general clinical situations. Each question was modified based on feedback from independent reviewers who were cardiovascular experts. Finally, the writing committee established four sections comprised of 95 clinical scenarios for various noninvasive modalities.

The appropriateness use criteria were defined with three ratings: appropriate (A), uncertain (U), and inappropriate (I). A questionnaire was emailed to the rating committee and then collected by the ASCI office after completion. The questionnaires were collected between December 2016 and February 2017 (22 of the 33 nominated members of the rating committee responded to the survey).

The questionnaire had four sections with 95 clinical scenarios. A total of two rounds of consensus survey were conducted; for each round, the appropriateness of utilization was categorized with a 9-point response scale: $1-3$ points as I, 4-6 points as $U$, and 7-9 points as A. For each round, different imaging modalities were separately scored for their appropriateness in a given scenario. When more than $50 \%$ of the panelists agreed on a category, the panel was considered to have reached a consensus for that particular clinical scenario. The questionnaire form included appropriateness criteria from other guidelines for each category and each noninvasive test modality [exercise ECG, echocardiography, positron emission tomography, single-photon emis- 
sion computed tomography (SPECT), RNI, CMR, and CCTA], the 9-point response scale, and space for additional comments. In the second round, the median scores from the previous round and the scores originally given by the answering panelist were shown for the questions for which agreement had not been reached. The questions with agreement reached in the previous round were not shown in the following round.

Of the 95 clinical scenarios-comprised of a total of 455 questions for different modalities-sent for the first round, consensus was reached on all modalities in 42 scenarios (197 questions). Of the other 53 scenarios (258 questions), 86 questions for which consensus was not reached were sent for the second round. The results of the consensus voting are included in the Supplementary (in the online-only Data Supplement).

When interpreting the score, several specific assumptions should be considered. Presumably, each test is performed in compliance with published criteria for quality cardiac diagnostic testing, locally available, and interpreted by experts who are qualified to do so. For exercise ECG, it should be assumed that the patient can exercise to a symptomatic endpoint or $85 \%$ of their age-predicted maximal heart rate. For echocardiography, SPECT, and CMR in evaluation of coronary artery disease, it is assumed that pharmacological stress test is performed to identify the presence of myocardial ischemia. Each modality has inherent risks such as radiation exposure, contrast sensitivity, and interpretation error. It is assumed that each modality should be chosen after weighing the risks and benefits in the specific clinical scenario. It should be assumed that CCTA and SPECT are performed using contemporary dose-saving techniques conforming to the As Low As Reasonably Achievable (ALARA) principle. For reasonable use of cardiovascular modality, the As High As Reasonably Achievable (AHARA) principle was considered.

The review committee, consisting of past-presidents, vice-presidents, and congress presidents, reviewed the AUC selected by consensus.

The development of the current AUC was funded by the ASCI.
However, the activities of the writing committee, the rating committee, and the review committee were independent of one another, and none of the three committees were influenced in any way by any of the funding for guideline development.

These guidelines should be revised as needed, following advances in technology, changes in the healthcare environment, and further accumulation of scientific evidence.

\section{RESULTS}

The final AUC ratings for multimodality cardiac imaging are divided into four sections and listed by clinical scenarios sequentially (Table 1-17).

\section{Section 1}

Detection of CAD: symptomatic or asymptomatic (Table 1-4).

\section{Section 2}

Cardiac evaluation in various clinical scenarios (Table 5-9).

\section{Section 3}

Use of imaging modality according to prior testing (Table 10,11 , and 12).

\section{Section 4}

Evaluation of cardiac structure and function (Table 13-17).

\section{DISCUSSION}

These new ASCI multimodality AUC were developed in order to reflect the current status of noninvasive cardiac imaging in Asia. In the current document, we present a synthesis of clinical experience for all commonly employed noninvasive imaging procedures for diagnosis of various cardiovascular diseases.

This document covers the same or similar clinical scenarios as the prior ASCI AUC for CT and MR and other modality guidelines for individual procedures. Overall, the AUC ratings for CT

Table 1. Symptomatic: non-acute chest pain suspected of stable coronary artery disease

\begin{tabular}{|c|c|c|c|c|c|}
\hline \multicolumn{6}{|c|}{ 1-1. Symptomatic: non-acute chest pain suspected of stable coronary artery disease } \\
\hline & Exercise ECG & $\mathrm{ECHO}$ & SPECT & CMR & CCTA \\
\hline \multicolumn{6}{|l|}{ ECG interpretable AND able to exercise } \\
\hline 1. Low pretest probability & A & I & I & I & $\mathrm{U}$ \\
\hline 2. Intermediate pretest probability & A & A & A & A & A \\
\hline 3. High pretest probability & A & A & A & A & A \\
\hline \multicolumn{6}{|c|}{ ECG uninterpretable OR unable to exercise } \\
\hline 4. Low pretest probability & I & $\mathrm{U}$ & $\mathrm{U}$ & $\mathrm{U}$ & A \\
\hline 5. Intermediate pretest probability & I & A & A & A & A \\
\hline 6. High pretest probability & I & A & A & A & A \\
\hline
\end{tabular}

ECG: exercise electrocardiography, SPECT: single-photon emission computed tomography, CMR: cardiac magnetic resonance, CCTA: cardiac computed tomography angiography, A: appropriate, U: uncertain, I: inappropriate 
are higher than those of the previous guidelines. This difference might be attributable to advances in CT technology, which have resulted in reduced radiation exposure and more accurate eval- uation of small structures with improvement in temporal resolution. In addition, wide availability of CT in Asian countries compared to CMR, which is less accessible, could be another

Table 2. Symptomatic: acute chest pain suspected of acute coronary syndrome

1-2. Symptomatic: acute chest pain suspected of acute coronary syndrome

Normal ECG AND cardiac biomarker

Exercise ECG ECHO SPECT CMR CCTA

7. Low global CAD risk

$\begin{array}{lllll}\text { U } & \text { A } & \text { A } & \text { U } & \text { A } \\ \text { U } & \text { A } & \text { A } & \text { U } & \text { A } \\ \text { I } & \text { A } & \text { A } & \text { U } & \text { A }\end{array}$

8. Intermediate global CAD risk

9. High global CAD risk

A

Non-diagnostic ECG OR equivocal cardiac biomarker

\begin{tabular}{|c|c|c|c|}
\hline 10. Low global CAD risk & I & A & \\
\hline 11. Intermediate global CAD risk & I & A & \\
\hline 12. High global CAD risk & I & A & \\
\hline 13. Abnormal ECG (ischemic nature) & $\mathrm{I}$ & A & \\
\hline 14. Acute chest pain of uncertain cause in emergency department ("triple rule out") & I & A & \\
\hline
\end{tabular}

ECG: exercise electrocardiography, SPECT: single-photon emission computed tomography, CMR: cardiac magnetic resonance, CCTA: cardiac computed tomography angiography, A: appropriate, U: uncertain, I: inappropriate, CAD: coronary artery disease

Table 3. Asymptomatic (1)

\begin{tabular}{|c|c|c|c|c|c|c|}
\hline \multicolumn{7}{|c|}{ 1-3. Asymptomatic (1) } \\
\hline & Exercise ECG & $\mathrm{ECHO}$ & SPECT & CMR & CAC & CCTA \\
\hline \multicolumn{7}{|l|}{ Framingham CHD risk } \\
\hline 15. Low & I & I & I & I & I & I \\
\hline 16. Intermediate & $\mathrm{U}$ & I & I & I & $\mathrm{U}$ & $\mathrm{U}$ \\
\hline 17. High & A & $\mathrm{U}$ & $\mathrm{U}$ & $\mathrm{U}$ & $\mathrm{U}$ & A \\
\hline \multicolumn{7}{|l|}{ Abnormal or uncertain prior testing } \\
\hline 18. Abnormal rest ECG (potentially ischemic) & A & A & A & A & $\mathrm{U}$ & A \\
\hline 19. Abnormal prior exercise ECG test & $\mathrm{U}$ & A & A & A & A & A \\
\hline 20. Zero CAC $>5$ years ago & I & I & I & I & I & I \\
\hline \multicolumn{7}{|l|}{ Positive CAC > 2 years ago } \\
\hline 21. $\mathrm{CAC}<100$ & I & I & I & I & & $\mathrm{U}$ \\
\hline 22. CAC $100-400$ & $\mathrm{U}$ & I & $\mathrm{U}$ & I & & $\mathrm{A}$ \\
\hline 23. CAC 401-1000 & A & $\mathrm{U}$ & $\mathrm{A}$ & $\mathrm{U}$ & & $\mathrm{U}$ \\
\hline 24. $\mathrm{CAC}>1000$ & $\mathrm{~A}$ & $\mathrm{U}$ & $\mathrm{A}$ & $\mathrm{U}$ & & $\mathrm{U}$ \\
\hline 25. Abnormal prior stress SPECT & I & $\mathrm{U}$ & $\mathrm{U}$ & $\mathrm{U}$ & & A \\
\hline
\end{tabular}

ECG: exercise electrocardiography, SPECT: single-photon emission computed tomography, CMR: cardiac magnetic resonance, CCTA: cardiac computed tomography angiography, A: appropriate, U: uncertain, I: inappropriate, CHD: coronary heart disease, CAC: coronary artery calcification

Table 4. Asymptomatic (2): post-revascularization (PCl or CABG)

\begin{tabular}{|c|c|c|c|c|c|}
\hline \multicolumn{6}{|c|}{ 1-3. Asymptomatic (2): post-revascularization (PCI or CABG) } \\
\hline & Exercise ECG & $\mathrm{ECHO}$ & SPECT & CMR & CCTA \\
\hline \multicolumn{6}{|l|}{ Post-revascularization (PCI or CABG) } \\
\hline 26. Incomplete revascularization (additional revascularization feasible) & $\mathrm{U}$ & $\mathrm{U}$ & A & A & A \\
\hline 27. Prior left main coronary stent & $\mathrm{U}$ & $\mathrm{U}$ & $\mathrm{U}$ & $\mathrm{U}$ & A \\
\hline 28. $<5$ years after $\mathrm{CABG}$ & I & $\mathrm{U}$ & $\mathrm{U}$ & $\mathrm{U}$ & A \\
\hline 29. $\geq 5$ years after $\mathrm{CABG}$ & $\mathrm{U}$ & $\mathrm{U}$ & $\mathrm{U}$ & $\mathrm{U}$ & A \\
\hline 30. $<2$ years after $\mathrm{PCI}$ & I & I & I & I & A \\
\hline 31. $\leq 2$ years after PCI & $\mathrm{U}$ & $\mathrm{U}$ & $\mathrm{U}$ & $\mathrm{U}$ & A \\
\hline
\end{tabular}

ECG: exercise electrocardiography, SPECT: single-photon emission computed tomography, CMR: cardiac magnetic resonance, CCTA: cardiac computed tomography angiography, A: appropriate, U: uncertain, I: inappropriate, PCI: percutaneous coronary intervention, CABG: coronary artery bypass graft 
cause of the improved rating of CT.

These rating differences might also reflect the changing practice environment and evolution in cumulative clinical experience with these procedures, as well as maturation of the field since publication of the original documents.

These new AUC are intended to provide guidance for clinicians when choosing among available testing modalities for various cardiac diseases. Each test was rated individually for each sce-

Table 5. Newly developed or suspected heart failure

\begin{tabular}{|c|c|c|c|c|c|}
\hline \multicolumn{6}{|c|}{ 2-1. Newly developed or suspected heart failure } \\
\hline & $\mathrm{ECHO}$ & Stress ECHO & SPECT & CMR & CCT \\
\hline 32. Initial evaluation of cardiac structure and anatomy & A & $\mathrm{U}$ & I & A & A \\
\hline \multicolumn{6}{|l|}{ Evaluation of ischemic etiology } \\
\hline 33. Angina/ischemic equivalent symptom & $\mathrm{U}$ & A & A & A & A \\
\hline 34. WITHOUT angina/ischemic equivalent symptom & A & A & A & A & A \\
\hline 35. Evaluation of ventricular function & A & A & A & A & $\mathrm{U}$ \\
\hline 36. Evaluation of myocardiial viability (after ischemic etiology determined) & $\mathrm{U}$ & A & A & A & $\mathrm{U}$ \\
\hline
\end{tabular}

SPECT: single-photon emission computed tomography, CMR: cardiac magnetic resonance, A: appropriate, U: uncertain, I: inappropriate, CCT: cardiac CT

Table 6. Cardiac evaluation prior to surgery

\begin{tabular}{|c|c|c|c|c|c|}
\hline \multicolumn{6}{|c|}{ 2-2. Cardiac evaluation prior to surgery } \\
\hline & Exercise ECG & $\mathrm{ECHO}$ & SPECT & CMR & CCT \\
\hline 37. Moderate-to-good functional capacity ( $\geq 4$ METs) or no clinical risk factor & I & I & I & I & I \\
\hline \multicolumn{6}{|l|}{ Poor or unknown functional capacity ( $<4$ METs) } \\
\hline 38. Low-risk surgery & I & I & I & I & I \\
\hline 39. Intermediate-risk surgery & $\mathrm{U}$ & $\mathrm{U}$ & $\mathrm{U}$ & $\mathrm{U}$ & $\mathrm{U}$ \\
\hline \multicolumn{6}{|l|}{ High-risk surgery } \\
\hline 40. Vascular surgery & $\mathrm{U}$ & $\mathrm{A}$ & A & $\mathrm{A}$ & $\mathrm{A}$ \\
\hline 41. Non-coronary cardiac surgery & $\mathrm{U}$ & A & A & A & A \\
\hline 42. Kidney or liver transplant & $\mathrm{U}$ & $\mathrm{A}$ & A & $\mathrm{U}$ & $\mathrm{A}$ \\
\hline
\end{tabular}

ECG: exercise electrocardiography, SPECT: single-photon emission computed tomography, CMR: cardiac magnetic resonance, A: appropriate, U: uncertain, I: inappropriate, MET: metabolic equivalent of task, CCT: cardiac CT

Table 7. Evaluation of arrhythmia or syncope without ischemic etiology

\begin{tabular}{lccccc}
\hline \multicolumn{4}{c}{ 2-3. Evaluation of arrhythmia or syncope without ischemic etiology } & & \\
\hline & Exercise ECG & ECHO & SPECT & CMR & CCT \\
\hline 43. Initial evaluation of cardiac structure and anatomy & I & A & I & A & U \\
44. Evaluation of ventricular function & I & A & U & A & U \\
45. Evaluation of myocardial scar or fibrosis & I & A & A & A & U
\end{tabular}

ECG: exercise electrocardiography, SPECT: single-photon emission computed tomography, CMR: cardiac magnetic resonance, A: appropriate, U: uncertain, I: inappropriate, CCT: cardiac CT

Table 8. Coronary revascularization

\begin{tabular}{|c|c|c|c|c|c|}
\hline \multicolumn{6}{|c|}{ 2-4. Coronary revascularization } \\
\hline & Exercise ECG & $\mathrm{ECHO}$ & SPECT & CMR & CCT \\
\hline \multicolumn{6}{|l|}{ Before revascularization } \\
\hline $\begin{array}{l}\text { 46. Evaluation of complex lesions before PCI (i.e., chronic total occlusions, } \\
\text { bifurcation lesions) }\end{array}$ & I & $\mathrm{U}$ & $\mathrm{U}$ & $\mathrm{U}$ & A \\
\hline 47. Myocardial viability & I & $\mathrm{U}$ & A & A & $\mathrm{U}$ \\
\hline \multicolumn{6}{|l|}{ After revascularization } \\
\hline 48. Suspected post-PCI myocardial infarction & I & A & A & A & $\mathrm{U}$ \\
\hline 49. Suspected ischemic chest pain after coronary revascularization & $\mathrm{U}$ & $\mathrm{U}$ & A & A & A \\
\hline
\end{tabular}

ECG: exercise electrocardiography, SPECT: single-photon emission computed tomography, CMR: cardiac magnetic resonance, A: appropriate, U: uncertain, I: inappropriate, PCI: percutaneous coronary intervention, CCT: cardiac CT 
Table 9. Kawasaki disease

2-5. Kawasaki disease

$\begin{array}{lllll}\text { Exercise ECG } & \text { ECHO } & \text { SPECT } & \text { CMR } & \text { CCT }\end{array}$

Asymptomatic

50. No previous definitive test available

$\begin{array}{lllll}I & \text { I } & \text { I } & \text { U } & \text { A }\end{array}$

51. Previous tests documented coronary aneurysm/stenosis, for follow up

$\mathrm{U}$

U U A

A

Symptomatic

52. No previous definitive test available

U

A

A A A

53. Previous tests documented coronary aneurysm/stenosis, for follow up

$\mathrm{U}$

U A A A

ECG: exercise electrocardiography, SPECT: single-photon emission computed tomography, CMR: cardiac magnetic resonance, A: appropriate, U: uncertain, I: inappropriate, CCT: cardiac CT

Table 10. Prior exercise ECG

\begin{tabular}{|c|c|c|c|c|c|}
\hline \multicolumn{6}{|c|}{ 3.1. Prior exercise ECG } \\
\hline & ECHO & SPECT & CMR & Calcium scoring & CCTA \\
\hline \multicolumn{6}{|l|}{ Normal exercise ECG } \\
\hline 54. Stable symptom & I & I & I & I & $\mathrm{U}$ \\
\hline 55. New onset or worse symptom & $\mathrm{U}$ & A & $\mathrm{U}$ & I & A \\
\hline \multicolumn{6}{|c|}{ Equivocal- or uninterpretable exercise ECG } \\
\hline 56. Stable symptom & $\mathrm{U}$ & A & A & $\mathrm{U}$ & A \\
\hline 57. New onset or worse symptom & A & A & A & I & A \\
\hline \multicolumn{6}{|l|}{ Abnormal exercise ECG } \\
\hline 58. Stable symptom & A & A & A & $\mathrm{U}$ & A \\
\hline 59. New onset or worse symptom & A & A & A & I & A \\
\hline
\end{tabular}

ECG: exercise electrocardiography, SPECT: single-photon emission computed tomography, CMR: cardiac magnetic resonance, A: appropriate, U: uncertain, I: inappropriate

Table 11. Prior SPECT

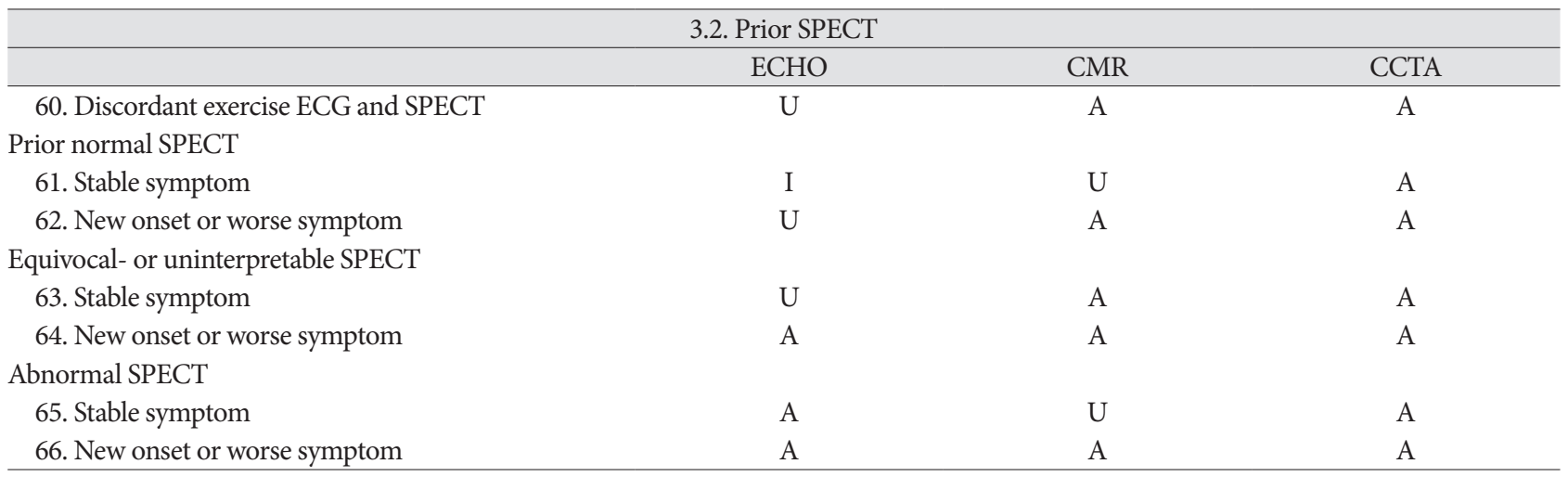

ECG: exercise electrocardiography, SPECT: single-photon emission computed tomography, CMR: cardiac magnetic resonance, A: appropriate, U: uncertain, I: inappropriate

Table 12. Prior CCTA

\begin{tabular}{lccc}
\hline & 3.3 Prior CCTA & & \\
\hline & ECHO & SPECT & CMR \\
\hline 67. Equivocal- or uninterpretable CCTA & $\mathrm{U}$ & $\mathrm{A}$ & $\mathrm{A}$ \\
Non-obstructive lesion & & $\mathrm{A}$ & $\mathrm{U}$ \\
68. Stable symptom & $\mathrm{U}$ & $\mathrm{A}$ & $\mathrm{A}$ \\
69. New onset or worse symptom & $\mathrm{A}$ & $\mathrm{A}$ & $\mathrm{A}$ \\
Obstructive lesion & & $\mathrm{A}$ & $\mathrm{A}$ \\
70. Stable symptom & $\mathrm{A}$ & $\mathrm{A}$ & \\
71. New onset or worse symptom & A & & \\
\hline
\end{tabular}

CCTA: cardiac computed tomography angiography, SPECT: single-photon emission computed tomography, CMR: cardiac magnetic resonance, A: appropriate, $\mathrm{U}$ : uncertain, I: inappropriate 
nario based on the quality of the published evidence as well as the expert opinion of the rating panel. In the absence of robust evidence of comparative effectiveness, a comparative rating ap- proach would be both premature and misleading. In addition, a larger number of radiologists in the writing and rating committees might have resulted in somewhat skewed ratings for certain

Table 13. Congenital heart disease

\begin{tabular}{|c|c|c|c|c|c|}
\hline \multicolumn{6}{|l|}{ 4-1. Congenital heart disease } \\
\hline & TTE & TEE & RNI & CMR & CCT \\
\hline 72. Evaluation of coronary anomaly & A & $\mathrm{U}$ & I & A & A \\
\hline 73. Assessment of complex congenital heart disease & A & A & I & A & A \\
\hline $\begin{array}{l}\text { 74. Anatomic assessment before percutaneous management of congenital heart disease } \\
\text { (ASD, PDA, etc.) }\end{array}$ & A & A & I & A & A \\
\hline 75. Assessment of post-operative congenital heart disease & A & A & I & A & A \\
\hline
\end{tabular}

RNI: radionuclide imaging, CMR: cardiac magnetic resonance, A: appropriate, U: uncertain, I: inappropriate, TTE: transthoracic echocardiography, TEE: transesophageal echocardiography, CCT: cardiac CT, ASD: atrial septal defect, PDA: patent ductus arteriosus

Table 14. Valvular heart disease (native valve AND prosthetic valve)

\begin{tabular}{|c|c|c|c|c|c|}
\hline \multicolumn{6}{|c|}{ 4-2. Valvular heart disease (native valve AND prosthetic valve) } \\
\hline & TTE & TEE & RNI & $\mathrm{CMR}$ & CT \\
\hline 76. Initial evaluation of valvular heart disease & A & A & I & A & $\mathrm{U}$ \\
\hline $\begin{array}{l}\text { 77. Evaluation of native cardiac valves } \\
\text {-Inadequate information from TTE }\end{array}$ & & A & I & A & A \\
\hline 78. Initial postoperative evaluation of prosthetic valve & A & A & I & $\mathrm{U}$ & $\mathrm{U}$ \\
\hline $\begin{array}{l}\text { 79. Evaluation of prosthetic cardiac valves } \\
\text {-Inadequate information from TTE }\end{array}$ & & A & I & A & A \\
\hline 80. Evaluation before transcatheter valve replacement & A & A & I & A & $\mathrm{A}$ \\
\hline
\end{tabular}

RNI: radionuclide imaging, CMR: cardiac magnetic resonance, A: appropriate, U: uncertain, I: inappropriate, TTE: transthoracic echocardiography, TEE: transesophageal echocardiography

Table 15. Cardiomyopathy (after ischemic etiology ruled out)

\begin{tabular}{lccccc}
\hline \multicolumn{4}{c}{ 4-3. Cardiomyopathy (after ischemic etiology ruled out) } & & \\
\hline & TTE & TEE & SPECT & CMR & CT \\
\hline 81. Suspected infiltrative cardiomyopathy & A & U & I & A & U \\
82. Suspected myocarditis & A & I & I & A & U \\
83. Suspected ARVD/C & A & A & I & A & U \\
84. Suspected cardiomyopathy due to cardiotoxic agent & A & I & I & A & U \\
85. Suspected hypertrophic cardiomyopathy & A & U & I & A & U \\
\hline
\end{tabular}

SPECT: single-photon emission computed tomography, CMR: cardiac magnetic resonance, CT: computed tomography, A: appropriate, U: uncertain, I: inappropriate, TTE: transthoracic echocardiography, TEE: transesophageal echocardiography, ARVD/C: arrhythmogenic right ventricular dysplasia/cardiomyopathy

Table 16. Electrophysiology study, ablation, ICD/CRT

\begin{tabular}{|c|c|c|c|c|c|}
\hline \multicolumn{6}{|c|}{ 4-4. Electrophysiology study, ablation, ICD/CRT } \\
\hline & TTE & TEE & SPECT & CMR & CT \\
\hline 86. Evaluation prior to RF ablation for $\mathrm{AF}$ & A & A & I & A & A \\
\hline \multicolumn{6}{|l|}{ Implantable cardioverter-defibrillator therapy } \\
\hline 87. Evaluation determine patient candidacy & A & A & I & A & A \\
\hline 88. Follow-up after placement & A & A & I & I & $\mathrm{U}$ \\
\hline \multicolumn{6}{|l|}{ Cardiac resynchronization therapy } \\
\hline 89. Evaluation determine patient candidacy & $\mathrm{A}$ & A & I & A & A \\
\hline 90. Follow-up after placement & A & A & I & I & $\mathrm{U}$ \\
\hline
\end{tabular}

SPECT: single-photon emission computed tomography, CMR: cardiac magnetic resonance, CT: computed tomography, A: appropriate, U: uncertain, I: inappropriate, TTE: transthoracic echocardiography, TEE: transesophageal echocardiography, RF: radiofrequency ablation, AF: atrial fibrillation 
Table 17. Cardiac mass, pericardial disease, and aorta

\begin{tabular}{|c|c|c|c|c|c|}
\hline \multicolumn{6}{|c|}{ 4-5. Cardiac mass, pericardial disease, and aorta } \\
\hline & TTE & TEE & PET & CMR & CT \\
\hline \multicolumn{6}{|l|}{ Mass } \\
\hline 91. Initial evaluation of suspected cardiac mass & A & A & I & A & A \\
\hline 92. Evaluation of cardiac mass, inadequate information from echocardiography & $\mathrm{U}$ & A & A & A & A \\
\hline \multicolumn{6}{|l|}{ Pericardial disease } \\
\hline 93. Initial evaluation of suspected pericardial disease & A & $\mathrm{U}$ & $\mathrm{U}$ & A & A \\
\hline 94. Evaluation of pericardial disease, inadequate information from echocardiography & $\mathrm{U}$ & $\mathrm{U}$ & $\mathrm{U}$ & A & A \\
\hline \multicolumn{6}{|l|}{ Aorta } \\
\hline 95. Evaluation of suspected aortic dissection, aneurysm, or inflammation & A & A & I & A & A \\
\hline
\end{tabular}

PET: positron emission tomography, CMR: cardiac magnetic resonance, CT: computed tomography, A: appropriate, U: uncertain, I: inappropriate, TTE: transthoracic echocardiography, TEE: transesophageal echocardiography

modalities. Thus, although these ratings reflect existing evidencebased practice supplemented by expert consensus, further research is needed to identify not only when to use any given modality, but also when to favor one over another.

\section{Supplementary Materials}

The online-only Data Supplement is available with this article at https://doi. org/10.22468/cvia.2017.00066.

\section{Conflicts of Interest}

The authors declare that they have no conflict of interest.

\section{REFERENCES}

1. Hendel RC, Patel MR, Kramer CM, Poon M, Hendel RC, Carr JC, et al. ACCF/ACR/SCCT/SCMR/ASNC/NASCI/SCAI/SIR 2006 appropriateness criteria for cardiac computed tomography and cardiac magnetic resonance imaging: a report of the American College of Cardiology Foundation Quality Strategic Directions Committee Appropriateness Criteria Working Group, American College of Radiology, Society of Cardiovascular Computed Tomography, Society for Cardiovascular Magnetic Resonance, American Society of Nuclear Cardiology, North American Society for Cardiac Imaging, Society for Cardiovascular Angiography and Interventions, and Society of Interventional Radiology. J Am Coll Cardiol 2006;48:1475-1497.

2. Taylor AJ, Cerqueira M, Hodgson JM, Mark D, Min J, O'Gara P, et al. ACCF/SCCT/ACR/AHA/ASE/ASNC/NASCI/SCAI/SCMR 2010 Appropriate Use Criteria for Cardiac Computed Tomography. A Report of the American College of Cardiology Foundation Appropriate Use Criteria Task Force, the Society of Cardiovascular Computed Tomography, the American College of Radiology, the American Heart Association, the American Society of Echocardiography, the American Society of Nuclear Cardiology, the North American Society for Cardiovascular Imaging, the Society for Cardiovascular Angiography and Interventions, and the Society for Cardiovascular Magnetic Resonance. J Cardiovasc Comput Tomogr 2010;4:407.e1-e33.

3. ASCI CCT \& CMR Guideline Working Group, Tsai IC, Choi BW, Chan C, Jinzaki M, Kitagawa K, et al. ASCI 2010 appropriateness criteria for cardiac computed tomography: a report of the Asian Society of Cardiovascular Imaging Cardiac Computed Tomography and Cardiac Magnetic Resonance Imaging Guideline Working Group. Int J Cardiovasc Imaging 2010; 26 Suppl 1:1-15.

4. Kim YJ, Yong HS, Kim SM, Kim JA, Yang DH, Hong YJ. Korean guidelines for the appropriate use of cardiac CT. Korean J Radiol 2015;16:251-285.

5. ASCI CCT and CMR Guideline Working Group, Kitagawa K, Choi BW,
Chan C, Jinzaki M, Tsai IC, et al. ASCI 2010 appropriateness criteria for cardiac magnetic resonance imaging: a report of the Asian Society of Cardiovascular Imaging cardiac computed tomography and cardiac magnetic resonance imaging guideline working group. Int J Cardiovasc Imaging 2010;26(Suppl 2):173-186.

6. Yoon YE, Hong YJ, Kim HK, Kim JA, Na JO, Yang DH, et al. 2014 Korean guidelines for appropriate utilization of cardiovascular magnetic resonance imaging: a joint report of the Korean Society of Cardiology and the Korean Society of Radiology. Korean J Radiol 2014;15:659-688.

7. Hendel RC, Berman DS, Di Carli MF, Heidenreich PA, Henkin RE, Pellikka PA, et al. ACCF/ASNC/ACR/AHA/ASE/SCCT/SCMR/SNM 2009 Appropriate use criteria for cardiac radionuclide imaging: a report of the American College of Cardiology Foundation Appropriate Use Criteria Task Force, the American Society of Nuclear Cardiology, the American College of Radiology, the American Heart Association, the American Society of Echocardiography, the Society of Cardiovascular Computed Tomography, the Society for Cardiovascular Magnetic Resonance, and the Society of Nuclear Medicine. J Am Coll Cardiol 2009;53:2201-2229.

8. American College of Cardiology Foundation Appropriate Use Criteria Task Force, American Society of Echocardiography, American Heart Association, American Society of Nuclear Cardiology, Heart Failure Society of America, Heart Rhythm Society, et al. ACCF/ASE/AHA/ASNC/HFSA/ HRS/SCAI/SCCM/SCCT/SCMR 2011 Appropriate Use Criteria for Echocardiography. A Report of the American College of Cardiology Foundation Appropriate Use Criteria Task Force, American Society of Echocardiography, American Heart Association, American Society of Nuclear Cardiology, Heart Failure Society of America, Heart Rhythm Society, Society for Cardiovascular Angiography and Interventions, Society of Critical Care Medicine, Society of Cardiovascular Computed Tomography, and Society for Cardiovascular Magnetic Resonance Endorsed by the American College of Chest Physicians. J Am Coll Cardiol 2011;57:11261166.

9. Patel MR, White RD, Abbara S, Bluemke DA, Herfkens RJ, Picard M, et al. 2013 ACCF/ACR/ASE/ASNC/SCCT/SCMR appropriate utilization of cardiovascular imaging in heart failure: a joint report of the American College of Radiology Appropriateness Criteria Committee and the American College of Cardiology Foundation Appropriate Use Criteria Task Force. J Am Coll Cardiol 2013;61:2207-2231.

10. White RD, Patel MR, Abbara S, Bluemke DA, Herfkens RJ, Picard M, et al. 2013 ACCF/ACR/ASE/ASNC/SCCT/SCMR appropriate utilization of cardiovascular imaging in heart failure: an executive summary: a joint report of the ACR Appropriateness Criteria ${ }^{\circledR}$ Committee and the ACCF Appropriate Use Criteria Task Force. J Am Coll Radiol 2013;10:493-500.

11. Gersh BJ, Maron BJ, Bonow RO, Dearani JA, Fifer MA, Link MS, et al. 2011 ACCF/AHA Guideline for the diagnosis and treatment of hypertrophic cardiomyopathy: a report of the American College of Cardiology Foundation/American Heart Association Task Force on Practice Guide- 
lines. Developed in Collaboration with the American Association for Thoracic Surgery, American Society of Echocardiography, American Society of Nuclear Cardiology, Heart Failure Society of America, Heart Rhythm Society, Society for Cardiovascular Angiography and Interventions, and Society of Thoracic Surgeons. J Am Coll Cardiol 2011;58:e212e260.

12. Fihn SD, Gardin JM, Abrams J, Berra K, Blankenship JC, Dallas AP, et al. 2012 ACCF/AHA/ACP/AATS/PCNA/SCAI/STS guideline for the diagnosis and management of patients with stable ischemic heart disease: a report of the American College of Cardiology Foundation/American Heart Association Task Force on Practice Guidelines, and the American College of Physicians, American Association for Thoracic Surgery, Preventive Cardiovascular Nurses Association, Society for Cardiovascular
Angiography and Interventions, and Society of Thoracic Surgeons. J AM Coll Cardiol 2012;60:e444-e164.

13. Wolk MJ, Bailey SR, Doherty JU, Douglas PS, Hendel RC, Kramer CM, et al. ACCF/AHA/ASE/ASNC/HFSA/HRS/SCAI/SCCT/SCMR/STS 2013 multimodality appropriate use criteria for the detection and risk assessment of stable ischemic heart disease: a report of the American College of Cardiology Foundation Appropriate Use Criteria Task Force, American Heart Association, American Society of Echocardiography, American Society of Nuclear Cardiology, Heart Failure Society of America, Heart Rhythm Society, Society for Cardiovascular Angiography and Interventions, Society of Cardiovascular Computed Tomography, Society for Cardiovascular Magnetic Resonance, and Society of Thoracic Surgeons. J Am Coll Cardiol 2014;63:380-406. 